\title{
CLASSIFICATION OF THE REAL INFINITE SIMPLE AND REAL INFINITE PRIMITIVE LIE ALGEBRAS
}

\section{By Steven Shnider}

H.ARVARD UNIVERSITY, CAMBRIDGE, MASSACHUSETTS

Communicated by Andrew M. Gleason, December 6, 1968

Abstract.-In this paper the real infinite simple and real infinite primitive Lie algebras are classified.

The results outlined in this paper conclude the classification of the transitive infinite Lie algebras of primitive type over the real types. These objects are introduced in [3]. In [1, 2] the complex primitive transitive infinite Lie algebras are classified through a study of the related pseudogroups. We use that classification and some related theorems of Guillemin and Sternberg to classify the real primitive transitive infinite Lie algebras.

The two relevant theorems are:

Theorem 1 (Guillemin). Any primitive transitive infinite Lie algebra $\left(L, L^{0}\right)$ contains a closed idea $I$ of finite codimension such that $\left(I, I \cap L^{0}\right)$ is simple and primitive.

TheOREm 2. Suppose $\left(M, M^{0}\right)$ is a real simple primitive transitive infinite Lie algebras. Let $L=M \otimes \mathbf{C}$ and $L^{0}=M^{0} \otimes \mathbf{C}$. If $L$ is simple then $\left(L, L^{0}\right)$ is primitive.

The classification proceeds as follows. Given $\left(L, L^{0}\right)$ a real primitive transitive infinite Lie algebra, let $I$ be the ideal given by the first theorem. Then $\left(I, I \cap L^{0}\right)$ is real primitive and simple. If $I \otimes \mathbf{C}$ is not simple then one can show that $I$ has already a complex structure. $\left(L, L^{0}\right)$ is contained in the transitive infinite Lie algebra of filtered derivations of $\left(I, I \cap L^{0}\right)$. We know that $\left(I, I \cap L^{0}\right)$ must be one of four possibilities. In this case the problem is reduced to determining the derivations of these four algebras, which is a quick computation.

If $I \otimes \mathbf{C}$ is simple then $\left(I \otimes \mathbf{C},\left(I \cap L^{0}\right) \otimes \mathbf{C}\right)$ is a complex primitive simple transitive infinite Lie algebras. We then proceed to classify the real forms. This is accomplished by looking at the corresponding graded algebra of the complex algebra and classifying the real forms of $g^{0}$ (S) $g^{-1}$. If $I \otimes \mathbf{C}$ is the algebra of all formal vector fields on $V=g^{-1}$ then $g^{0}=g l(V, \mathbf{C})$. In this case all real forms are of the form $g l(W, \mathbf{R})$ (S) $W$, where $W$ is a real form for $V$. If $I \otimes \mathbf{C}$ is all formal vector fields of divergence 0 then $g^{0}$ (S) $g^{-1}=\operatorname{sl}(V, \mathrm{C})$ (S) $V$ and all real forms are of the form $\operatorname{sl}(W, \mathbf{R})$ (S) $W$, where $W$ is a real form of $V$. If $I \otimes \mathrm{C}$ is all Hamiltonian vector fields then $g^{0}$ (S) $g^{-1}=s p(V, \mathrm{C}, J)$ (S) $V$, where $s p(V, \mathrm{C}, J)$ is the complex symplectic algebra relative to the skew symmetric bilinear form $J$. In this case all real forms are of the form $s p\left(W, I R, J^{\prime}\right) W$, where $W$ is a real form of $V$ and $J^{\prime}$ is a skew symmetric real bilinear form on $W$. If $I \otimes \mathbf{C}$ is the contact algebra then if $g^{-1}=V=V_{0} \oplus\{z\} g^{0}=\operatorname{sp}\left(U_{0}, \mathbf{C}, J\right) \oplus$ $\{I\}$ (S) $U_{0}$, where $U_{0}=V_{0} \otimes z^{*}$. All real forms are of the form

$$
g^{\prime \prime}=\operatorname{sp}\left(Y_{0}, \mathrm{R}, J^{\prime}\right) \oplus\{I\} \subseteq \underset{466}{Y_{0}} \quad g^{-1}=W=W_{0} \oplus\{z\}
$$


where $Y_{0}=W_{0} \otimes \bar{z}^{*}$ and $W$ is a real form of $V$.

Since all four complex algebras $I$ (S) $\mathrm{C}$ have $g^{k}=$ the $k^{\text {th }}$ prolongation of $g^{0}$, by a dimension argument we conclude that the higher terms in the graded algebra of $I$ are the full prolongations. Hence we have determined the graded structure of I. In order to determine the filtered structure we must study the cohomology groups. All four possibilities for $I$ are involutive hence the higher cohomology groups are zero. There are no invariants in $H^{-1,2}\left(g_{I}\right)$ for $g_{I}=\operatorname{Gr}(g l(W))$, $\operatorname{Gr}(s l(W)), G r(s p(W))$. If $g_{I}$ is the last of the four possibilities $H^{-1,2}\left(g_{I}\right)$ is one dimensional which means there are two possible filtered algebras giving the graded algebra $g_{I}$. This means that there is a unique transitive infinite Lie algebra which is not flat and has the graded structure of $g_{I}$. Since $I \otimes \mathrm{C}$ is not flat, $I$ is not flat and hence its structure is determined.

To find $\left(L, L^{0}\right)$ we must determine the filtered derivations of $\left(I, I \cap L^{0}\right)$. $\left(L, L^{0}\right)$ lies between $\left(I, I \cap L^{0}\right)$ and this algebra of derivations. A quick computation determines the latter algebra and we conclude that $\left(L, L^{0}\right)$ must be among the following:

(1) The algebra of all real formal vector fields on $W$,

(2) The algebra of all real formal vector fields on $W$ with divergence 0 ,

(3) The algebra of all real formal vector fields on $W$ with constant divergence,

(4) The algebra of all real Hamiltonian vector fields on $W$,

(5) The algebra of all real formal vector fields on $W$ preserving a Hamiltonian

form up to a constant multiple, and

(6) The real contact algebra.

From the case when $\left(I, I \cap L^{0}\right)$ already has a complex structure we find the following possibilities for $\left(L, L^{0}\right)$ :

(1) The algebra of all complex formal vector fields on $V$,

(2) The algebra of all complex formal vector fields on $V$ with divergence 0 ,

(3) The algebra of all complex formal vector fields on $V$ with divergence on a ray in $\mathbf{C}$,

(4) The algebra of all complex formal vector fields on $V$ with constant divergence,

(5) The algebra of all complex formal Hamiltonian vector fields on $V$,

(6) The algebra of all complex formal vector fields on $V$ preserving a Hamiltonian form up to a real multiple of $e^{\theta}$ for some fixed $\theta$,

(7) The algebra of complex formal vector fields preserving a Hamiltonian form up to a complex constant multiple, and

(8) The complex contact algebra.

1 Guillemin, V., D. Quillen, and S. Sternberg, "Classification of irreducible complex algebras of infinite type," Journal d'Analyse Mathematique, 18 (1967).

${ }^{2}$ Guillemin, V., D. Quillen, and S. Sternberg, "Classification of complex primitive infinite pseudogroups," these Procendings, 55, 687 (1966).

${ }^{3}$ Guillemin, V., and S. Sternberg, "Transitive Differential Geometry," Bulletin of A.M.S., 70 (1964). 